\title{
Psychological Problems and Intervention of College Students under New Media
}

\author{
Jian Jie \\ Chongqing Electronic Engineering, Chongqing, Shapingba, 401331, China \\ jianjie_tx@126.com
}

Keywords: New media, College students, Psychological intervention.

\begin{abstract}
Under the new media era, personal consciousness of college students gets enhanced, and their thought tends to be diverse. Besides, communication, sharing and expression habits also become different as before. If college students can not correctly understand and treat new media, it is quite easy for them to have psychological dependence and psychological problems. Therefore, educators should enhance their understanding of new media, optimize communication among them, share environment of new media and strengthen construction of new media to combine schools, families and society together and guide them to correctly treat new media which will give assistance for their physical and psychological development.

New media refers to a kind of new media form under the support of new technology, such as digital newspaper, digital magazine, digital TV, digital radio, mobile phone message, network, touch media, etc. Compared with the four traditional media newspaper, outdoors, radio and TV, new media is vividly called "the fifth media". According to the 31 th 《Statistic Report on the Development of China Internet Network》 released by CNNIC in January2013, the number of Chinese netizens reached to 564 millions, internet penetration $42.1 \%$, mobile phone netizens 420 millions, weibo ranging from 63.11 millions in 2010 to 309 millions by the end of 2013 with its users' ratio reaching to $54.7 \%$.

New media has become an important way for college students to broaden their knowledge, while demand on new media for college students has a close correlation with their physical and psychological development. Therefore, how to cope with challenges from new media and carry out physical and psychological education on college students becomes critically significant. In the "Exchange Exhibition of the League New Media and Cultural Achievements in 2012" and "the 6th Development Forum of Chinese College Students in 2010", both Lu Hao and Wang Hongyan pointed that league organizations should actively meet the challenges of new media and well use new media as culture carrier. Therefore, it becomes the focus and problem to be solved that educators should deeply observe the effects of new media on college students to make it well promote the healthy growth of college students.
\end{abstract}

\section{Psychological problems of college students caused by new media}

Under the new media environment, people are easy to lose themselves especially facing negative information due to the openness and compatibility of new media, and it will cause peoples' psychological changes if it is not handled properly. For college students at the stage of psychological development, they are more susceptible to the effects, and the healthy growth will be affected even it will bring damages to society if new media information is not well dealt with when it cause psychological changes for them.

Effect on formation of correct values and world views of college students. The openness of new media makes information spread under barrier-free condition, and college students are free to accept different points of view, among which are some adverse social opinions that will seriously have influence on the formation of college students' values and cause the missing of value orientation. Ways for college students to accept new media information tend to be passive, perceptive, irrational and lack of initiative which will lead to the weakness of thinking ability and in converse it will do harm to their growth and development. 
Causing moral loss. The concealment of new media, especially indirect communication as its language communication together with the virtuality of network identity likely cause some criminal activities. Behind new media, there exist many hacker attacks, network frauds, etc. For youth college students, it is very easy to cause their behavioral disorders, psychological dislocation even some will explore unmoral behaviors which will finally lead to loss of moral emotion if they just pursue the perfect of themselves and can nor realize the conversion between reality and network virtual characters. Facing new media, moral judgment and moral of college students will also face new challenges. Due to the inexactitude in filtering of new media, some useful and useless information appears. However, college students can build their own room through the single mode of receiving information adopted by new media, but it will lead them to develop mental paralysis disease, the mainstream moral pursuit will be drowned by personal pursuit and cause the loss of moral judgment. Thus moral judgment ability of college students will face challenges under the diverse situation of new media environment ethics.

Virtualization of friendship and love as well as dependence on new media. Network friendship and love of new media are virtual, and they are extension of network society. In fact, friendship and love in reality have big difference with that of network. True feelings can be infused in friendship and love in real life only when people are fully familiar with each other and trust each other in communication, which is also a process of cognition and development. Friendship and love bear burdens of social obligations and responsibilities. After long-term addiction to virtual network emotion and blindly treating emotion, college students will suffer a lot from emotion problems. After long-term addiction to online games, chats, etc, students will become dependent on new media, and they can not live a normal life once they become addicted to them, thus their physical and psychological development will be badly hurt.

\section{Causes of Psychological Problems from New Media}

The intrinsic characteristic of new media is an important condition causing psychological problems of college students. New media is generated on the basis of network communication, and it has characteristics of openness, virtuality, informative, etc. which are enough to make immature college students dazzled. The open new media and its large participation are key points that make new media environment complex, and it is easy to make college students have psychological identification with virtuality of new media because they can constantly change their identities, break up traditional space-time block, ignore reality and create a complete virtual environment that cause confusions between virtuality and reality. Besides, new media offers college students large information which will cause indigestion, even some students with lower will become addicted to this convention and dependent on it, and finally they can not escape from it.

Some adverse social factors are main causes of psychological problems of college students. College students are at the stage of immature psychological development. They childishly understand and deal with some things, and are also easy to treat things emotionally in attitudes and methods. As the fastest medium reflecting social affairs, new media is directly connected with reality, and it makes it more easier for college students to suffer damages from social information. Some virtual and adverse information easily causes their impulse and leads to psychological problems which will have negative effects on their growth. Besides, teenagers have a lack of calmness and rationality because of the vulnerability of their psychological bearing ability, and their psychological performance turn to be vulnerable which will makes them depressed and passive in dealing with things. New media environment has a significant effect on psychological development fo college students, so good social environment is a key factor for healthy growth of college students.

Psychological contradiction is the root of psychological problems for college students. There is no problem of new media itself, but psychological problems of college students under new media environment are caused by their own problems. College student are at the critical stage of psychological development. Firstly, communication demand and psychological closeness are in paradox. Mental and emotional demands of college students increase, and their communication 
demand increase correspondingly, and in converse their contradiction with psychological closure becomes bigger and bigger. Secondly, it is the contraction between knowledge desire and identification ability. When college students want to explore every field of social life, there will have conflict between their knowledge desire and identification ability due to their limited knowledge and experience, and this conflict will decrease their confidence. Thirdly, it is the contradiction between sense and reason. College students are at the sensitive stage of physical and psychological development, and they hope to deal with things sensibly but in fact it is hard for them to control their emotions and they will act impetuously. When they can not release depression in their mind, a series of psychological problems will appear. Therefore, psychological contradiction is the root of their psychological problems.

Full play of educational resultant force under new media is an important cause of psychological problems for college students. Educational resultant force refers that schools, families and society interact with each other, coordinate with each other and communicate with each other to form the force with school education as main body, family education as basis and social education as rely to improve the actual effect of educational activities after making them integrated. It is pointed out in 《Ideological and Political Education Methodology》that the so-called educational resultant force is the comprehensive effect after implanting comprehensive education at a certain time and a certain condition.

\section{Strategies of psychological problems of college students under new media}

To establish a sound management mechanism. Firstly, it is to increase the intensity of network legislation. The relevant state departments should establish and perfect network legislation according to development and management demand to provide legal protection for standardization of new media. Secondly, it is to strengthen the consciousness of law and regulate the new media behaviors of college students. Rapid spread and broad range are characteristics of new media. If rapid spread of new media events especially emergencies can be dealt with properly, it can not cause bring about great influences, but it they are not dealt with timely, adverse consequences will be generated. Therefore, it is critically important to establish early warning mechanism and enhance people's legal consciousness. Finally, relevant functional departments should strengthen researches on direction of new media, explore new mechanism of network management, grasp development trend of new media and timely observe college students' thought through network forum, blogs, microblogging, QQ and other instant communication ways to provide theoretical basis for targeted education plans. New media technology develops rapidly, and the management departments should also follow the new trend of development to actively explore new ways to manage new media and build good environment for the healthy development of college students.

To optimize new media environment. New media provides a convenient way for college students to acquire knowledge, and it also promote the initiative for them to acquire knowledge which changes their ways of communication, sharing and expression to some extent. Under new media environment, information tend to be diverse and complex, and college students even their communication and expression ways are likely to be disturbed when they are at this special stage. Therefore, educators should pay more attention to college students' scientific and rigorous attitudes to avoid adverse effects. Whether new media can serve for college students' healthy growth is mainly dominated by leading mechanism. Management departments at all levels should establish educational new media that are various in form and rich in content to satisfy college students' real life. To enrich college students' practical life, integration of network and real life should be actively promoted and network emotional alienation phenomenon should be prevented to develop positive and healthy network culture.

To change education ideas and strengthen psychological health education under new media. Under new media environment, way of participation and expression for college students are more free, and network behaviors are more diverse. Thus it puts forward more demand on education. Therefore, our education must be people-oriented, advance with times, change ideas, seriously take 
a look at traditional management modes and concepts and perfect education system of college students. Firstly, educators should understand features and regulations of new media, improve their own quality, actively participate in new media, improve their ability in using and masting new media, and develop and implement targeted education activities according to features of rapid spread and wide range of new media, for example, using new media to establish communication group is a way to satisfy educational demand for college students of all levels. Besides, psychological consulting rooms online can be established to provide consultation and guidance to ensure that college students can get timely and professional assistance.

\section{References}

[1] The 31th 《Statistic Report on the Development of China Internet Network》 released by CNNIChttp://news.xinhuanet.com/tech/2013-01/15/c_124233840.htm, 2013-01-15.

[2] Shi JIa. Introduction to New Media. CUCP Press, 2006:1-25.

[3] Wang Chuanzhong. Influence of New Media on Life, Study and Thought of College Student.Theoretical Front in Higher Education, 2009(7).

[4] Zhang Junhui. Influence of New Media on College Students and Education Strategies .Journal of Anyang Normal University( social science edition), 2012(2) 141-144.

[5] Liu Yumei. Crisis and Reconstruction: Moral Identity Analysis of College Students Online.Journal of Chongqing University of Technology（philosophy and social science edition ), 2008(6).

[6] Zhao Min. Researches on Moral Education of College Students under New Media. Shandong University, 2012(3). 